\title{
Creating Public Health-Based Interventions to Address Sexuality as a Determinant of Health Disparities
}

\author{
Kittu Pannu* \\ Mailman School of Public Health, USA
}

Submission: October 02, 2018; Published: October 29, 2018

*Corresponding author: Kittu Pannu, Mailman School of Public Health, USA

\section{Mini Review}

Health disparities amongst sexual minorities are not a new phenomenon - research institutes have been calling for researchers to fill in the gaps in available materials for years. The list of diseases that disproportionately affect sexual minorities compared to their heterosexual counterparts increases as more research delves into this new area of study, including heart conditions, mental illnesses, substance abuse issues, and suicide [1] Evidence points to access, mistrust, stigma, and overall chronic stress as major contributors to these discrepancies in health outcomes. Studies have already linked the LG and B identities to higher levels of mental health issues, smoking, and activity limitation. Researchers believe that these issues may be due to an overall increase in stress experience for people identifying with these sexualities, a lack of healthy coping mechanisms, social isolation, and a lower overall quality of life [1] As public health practitioners, it is our duty to address and alleviate these issues in order to develop a higher quality of life for this vulnerable population. We can do so by directing more funding toward research efforts that delve into understanding which intervention styles can decrease these disparities and increase positive health outcomes for this vulnerable population.

Stigma is a major contributing factor to health disparities observed in minority populations, including sexual minorities. $[2,3]$ Through its chronic nature, stigma can increase one's daily psychological stress, leading to deleterious physical and mental health effects. Stigma impacts from a variety of areas including from within, from others, and from one's own environment and community. Researchershave concluded thatsexual minorities who live in communities that are known to create higher-stigmatizing environments tend to live shorter lives when compared to their peers in lower stigma-creating areas. Discrimination, prejudice, and marginalization within communities have the ability to shape stress levels and, ultimately, health outcomes for sexual minorities. Researchers have stated that these findings are in line with health outcomes due to minority stress experienced through race in the South [4] Minority groups within the umbrella of sexual minorities, such as those who identify as bisexual or who engage in homosexual activity without self-identifying as LGBT are at an elevated risk for negative health outcomes than their other LGBT counterparts, much of which researchers expect is due to a heightened level of stress surrounding that person's identity. In other words, if a person is a part of an even more marginalized group of a marginalized community, they tend to experience more negative health outcomes. Issues of hegemonic masculinity, or when men refuse to seek out medical care for their ailments as a means to remain self-sufficient, can further drive disparities amongst these already-marginalized groups.

Many of these disparities hinge on access and utilization issues, including timely utilization when issues arise [2,3] Sexual minorities have less access to healthcare services, which translates to fewer opportunities to take care of their overall health. Currently, researchers are trying to observe if specific sexual identity may further exacerbate these differences. Sexual minorities have reported higher levels of expected/experienced stress and barriers to healthcare, especially when they consider the expected and experienced discrimination felt from disclosing a homosexual identity to a healthcare professional. After interviewing healthcare staff members and patients, Everett and Mollborn correlate this negative reaction to a lack of cultural competency trainings available to staff members [3].

Everett and Mollborn have shown that sexual minorities are, like other minorities, not immune to hate crimes and other discriminating acts [5] Nearly 20 percent of sexual minorities stated that they had experienced criminal activity as a result of their orientation. Focusing on a perceived disparity between the genders, Everett and Mollborn attribute these findings to how men are victims of violent crime more so than women and how most perpetrators of these attacks are heterosexual men. This stigma can confer onto sexual minorities higher levels of psychological distress, opening them up to higher levels of mental health issues [5]. Increasing mental health outcomes in sexual minorities has been linked to help reduce healthcare needs, self-esteem, and confidence in the medical system to treat their physical ailments [3] Other interventionists have pointed to a lack of education-based interventions as a possible way to improve 


\section{Juniper Online Journal of Public Health}

health outcomes for this population. Hatzenbuehler highlighted how many LGBT population-targeting public health interventions focus on gay and bisexual men only, leaving out a substantial part of this underserved population. The authors recommend a focus on education to encourage potential patients to receive the care they need [3]. Unfortunately, current policy and governmental action have not made things any easier for sexual minorities. Whether they deny equal protections on a national level for LGBT people through the introduction of numerous religious exemption bills or those that prevent municipalities or counties from passing nondiscrimination protections, many politicians have attempted (and succeeded) to roll back protections through the law targeting this particular minority group. The issue athand is how we as public health practitioners can alleviate some of these issues and create an environment that is more hospitable to sexual minorities.

Based on this, I propose the following steps to alleviate some of the issues facing sexual minorities:

a) Increase the amount of effective cultural competency trainings for healthcare workers that will engage and shape their behaviors. Doing so will help to educate and familiarize healthcare workers on a culture they may not understand and can help in humanizing these patients. Cultural competency programs can also aid in teaching these workers how to provide healthcare to people whose lifestyles they may not agree with while still behaving in a professional and helpful manner. By doing so, we can help displace some of the stigmatizing behaviors in which some healthcare workers may engage and increase sexual minority trust in the healthcare system.

b) Introduce positive discussion on sexuality and related topics through sexual education curricula in middle and high schools and create mandatory training programs for sexual education instructors that address how to remove stigma from nontraditional sexual identities. By introducing this to teens and young adults, we can ensure that these topics will receive exposure. Addressing it in a positive manner can help to begin the process of dismantling certain aspects of structural stigma associated with being a sexual minority. Creating training programs can work to ensure that instructors are exposed to de-stigmatizing language that we would encourage them to use with their students.

c) Create more public health campaigns to target the minority groups within the sexual minority population. Focus on educating lesbians and bisexual women on the benefits of engaging the healthcare system. By doing so, public health interventionists can educate these vulnerable groups on the benefits of health screenings and proactive healthcare action. This can decrease health disparities between heterosexual and homosexual women.

d) Increase the amount of research projects dealing with these topics. This can help with understanding the extent to which stress, stigma, sexuality, healthcare access, mistrust of the medical system, and other related topics all contribute to health outcomes for sexual minorities.

As stated above, it is up to us to do something about these health disparities. We need to delve into this branch of research to expand and learn more about how these issues affect overall health outcomes, create evidence-based interventions to alleviate these issues, and then implement and continually evaluate them in the real world. Change may not occur immediately, but we can create the foundation for change that can shape the health outcomes of generations of sexual minorities to come.

\section{References}

1. US Department of Health and Human Services. Top Health Issues for LGBT Populations Information and Resource kit.

2. Fredriksen Goldsen KI, Kim H, Barkan SE, Muraco A, Hoy Ellis CP (2013) Health Disparities Among Lesbian Gay and Bisexual Older Adults: Results from a Population Based Study. American Journal of Public Health 103(10): 1802-1809.

3. Hatzenbuehler ML, Bellatorre A, Lee Y, Finch BK, Muennig P (2014) Structural stigma and all-cause mortality in sexual minority populations. Social Science \& Medicine 103: 33-41.

4. Herek GM (2008) Hate Crimes and Stigma-Related Experiences Among Sexual Minority Adults in the United States: Prevalence Estimates from a National Probability Sample. Journal of Interpersonal Violence, 24(1): 54-74.

5. Everett BG, Mollborn S (2013) Examining Sexual Orientation Disparities in Unmet Medical Needs Among Men and Women. Population Research and Policy Review 33(4): 553-577.

\section{Your next submission with Juniper Publishers will reach you the below assets}

